\title{
EEG Features in Adolescent Patients with Borderline and Narcissistic Personality Disorder
}

\section{Iznak AF*, Iznak EV, Damyanovich EV, Krylova ES, Kuleshov AA and Kaleda VG}

Mental Health Research Centre, Moscow, Russian Federation

*Corresponding Author: Iznak AF, Professor, Head of Laboratory of

Neurophysiology, Mental Health Research Centre, Moscow, Russian Federation.

DOI: 10.31080/ASNE.2022.05.0470
Received: November 15, 2021

Published: December 31, 2021

(C) All rights are reserved by Iznak AF., et al.

\section{Abstract}

Personality disorders (PD) in adolescence are widespread. It creates problems of social adaptation of patients and represents significant risk factors for auto-aggressive behavior, including suicidal one. The neurobiological basis and EEG markers of PD in adolescence have not been adequately studied. The aim of the study was identification of EEG features and their correlations with clinical parameters in adolescent patients with borderline personality disorder (BPD) and narcissistic personality disorder (NPD), possibly mediating some aspects of their clinical traits.

28 BPD patients (301.83, by DSM-5) and 24 NPD patients (301.81, by DSM-5), as well as 24 healthy controls (HC) aged 16-25 years were enrolled in the study. Clinical scales HDRS-21 and HAM-A were used for quantitative assessment of patient's conditions. Pretreatment background EEG was recorded, and EEG spectral analysis was carried out in 8 narrow frequency sub-bands. Descriptive statistics and correlation analysis of the set of EEG and clinical data were performed.

EEG spectral parameters in BPD did not differ significantly from those of HC. NPD shows the EEG signs of more activated brain cortex than in both BPD and norm caused by decreased functional state of the anterior cortical regions. The structure of correlations between EEG parameters and clinical scores also differed between BPD and NPD.

The data obtained suggests that these features of the functional organization of the brain activity may contribute to the disturbance of the processes of regulation of emotions and control of behavior in adolescent patients with BPD and NPD, more pronounced in NPD.

Keywords: Adolescence; Borderline Personality Disorder; Narcissistic Personality Disorder; Depression; Quantitative Electroencephalography

\section{Abbreviations}

EEG: Electroencephalography; PD: Personality Disorder; BPD: Borderline Personality Disorder; NPD: Narcissistic Personality Disorder; NSSI: Non-Suicidal Self-Injuries; MRI: Magnetic Resonance Imaging

\section{Introduction}

Differential diagnosis of borderline and narcissistic personality disorder (PD) in adolescence presents certain difficulties due to their belonging to the general demonstrative cluster of PDs, lack of formation of the main personality traits, polymorphism of clinical manifestations caused by the influence of the age factor. Borderline personality disorder (BPD) and narcissistic personality disorder (NPD) are common in adolescence. BPD in the general population occurs with a frequency of $1-3 \%$ [1], in outpatients - 11-33\%, [2], in hospitalized patients - up to 43-49\% [3]. The prevalence of NPD - in the general population is about $5 \%$, and among patients hospitalized due to suicidal activity it reaches $23 \%$ [4]. 
In addition to the problems of social adaptation of patients, these disorders are significant risk factors for auto-aggressive behavior, including suicidal one [5-8]. Up to $60-85 \%$ of patients with BPD commit non-suicidal self-injuries (NSSI) without the intention of taking their own life [9], and therefore NSSI is included in the list of diagnostic criteria for BPD [10]. In NPD, the prevalence of NSSI is somewhat lower (reaches almost 36\%) [11], but narcissistic individuals are at increased risk of suicide even in the absence of any psychopathological clinical manifestations [12]. This dictates the need to identify the neurobiological nature of the main psychopathological mechanisms of auto-aggression in adolescence in order to develop additional criteria for the differential diagnosis of different types of personal pathology during its formation.

Studies of the neurobiological bases and markers of BPD and NPD in adolescence are conducted in several directions [13]. Structural MRI methods have shown that in girls with BPD, compared with the norm, the volume of gray matter in the dorsolateral prefrontal cortex of both hemispheres and in the left orbitofrontal cortex is reduced $[13,14]$. In patients with NPD, the volume of gray matter in the fronto-paralimbic areas, including the rostral and middle cingulate gyrus, the anterior part of the left insula, and the dorsolateral and medial prefrontal cortex, is reduced [15].

Data on EEG correlates for BPD and NPD are often contradictory and more often refer to certain syndromes and/or behavioral deviations within these disorders. Thus, in patients with BPD, most EEG parameters do not differ from the norm [16]. In BPD, positive correlations were found between the EEG theta activity spectral power values and reduced pain sensitivity and self-harm [17], as well as with the severity of suicidal thoughts [18]. The predominance of the EEG alpha rhythm in the parietal leads is associated with excessive self-control, and predominance of the delta activity in the frontal regions is associated with insufficient self-control of behavior [19]. Special attention is paid to the interhemispheric asymmetry of the alpha rhythm of the background EEG in the frontal regions. At the same time, some authors consider EEG alpha frontal asymmetry as EEG marker of BPD [20], others do not find alpha asymmetry of the background EEG at rest in patients with BPD [21]. There were also found positive correlations of frontal alpha asymmetry with the severity of neuroticism and impairments in the assessment of facial expression [22].

Previously, we identified the features and differences in the frequency and spatial parameters of the EEG (the ratios of the spectral power of the frequency components of the alpha rhythm and the interhemispheric asymmetry of the alpha rhythm in the posterior EEG leads) in patients with NSSI and suicidal attempts [23,24]. In the present study, it was proposed, using similar methods of EEG analysis, to identify EEG features reflecting the differences in the functional organization of brain activity in BPD and NPD patients, possibly mediating some aspects of the clinical picture of the disorders.

The aim of the study was to identify the EEG features in patients with narcissistic and borderline personality disorder, possibly mediating some aspects of the clinical picture of the disorders.

\section{Materials and Methods}

The clinical-neurophysiological study had an open design and was carried out in compliance with the modern standards of biomedical ethics in the Laboratory of Neurophysiology and the Department of Youth Psychiatry of the Mental Health Research Centre (MHRC, Moscow, Russian Federation), and approved by local ethics committee of the MHRC.

Patients

52 male patients (all right-handed, aged 16-25 years) were enrolled in the study. The patients were on inpatient treatment or on outpatient observation in the Department of Youth Psychiatry of the MHRC clinic seeking for medical help due to depressive state. 28 patients with borderline personality disorder (BPD; mean age $20.4 \pm 3.0$ years old) and 24 patients with narcissistic personality disorder (NPD; mean age $20.5 \pm 3.4$ years old) consisted two experimental groups. To clarify the type of personality disorder, the Structured Clinical Interview for DSM-5 Personality Disorders (SCID-5-PD) [25] was used. 24 healthy volunteers matched by age and gender consisted control group (HC; mean age $20.3 \pm 3.2$ years old). The BPD, NPD and HC groups did not statistically differ in age and social-demographic parameters. The majority of subjects were students.

- Inclusion criteria for the study: Age from 16 to 25 years, inclusive; diagnoses: borderline personality disorder (301.83 by DSM-5; F60.3 by ICD-10) or narcissistic personality disorder (301.81 by DSM-5; F60.81 by ICD-10).

- Non-Inclusion criteria: Age under 16 and over 25; the presence of signs of the central nervous system organic disease or chronic somatic diseases in the stage of decompensation. 
According to the requirements of the World Medical Association's Declaration of Helsinki, all subjects signed an informed consent to participate in the study.

\section{Clinical assessments}

Quantitative clinical assessments of the pre-treatment depression severity were determined using the Hamilton's scale for depression (HDRS-21) [26]. The following scores were taken into account: total score of the HDRS-21 scale, the sum of depression cluster (the sum of points 1, 2, 3, 7 and 8 of HDRS-21 scale), the sum of anxiety cluster (the sum of points 9, 10 and 11 of HDRS21 scale), the sum of sleep disorders cluster (the sum of points 4, 5 and 6 of HDRS-21 scale), and the sum of ideational disorders cluster (the sum of points 15, 17, 19, 20 and 21 of HDRS-21 scale). Additionally, the pre-treatment anxiety severity was quantitatively determined using the Hamilton's scale for anxiety (HAM-A) [27].

\section{EEG registration and analysis}

All patients underwent multichannel (16 channels) registration of the background EEG in a state of quiet wakefulness with eyes closed before the start of the treatment course and in absence of previous medication. EEG was recorded in leads: F7, F3, F4, F8, T3, $\mathrm{C} 3, \mathrm{Cz}, \mathrm{C} 4, \mathrm{~T} 4, \mathrm{~T} 5, \mathrm{P} 3, \mathrm{Pz}, \mathrm{P} 4, \mathrm{~T} 6, \mathrm{O} 1$ and $\mathrm{02}$, according to the International 10-20 system, relative to joint ear-lobe referents $A 1+A 2$, using the "Neuro-KM" hardware-software equipment ("Statokin", Russian Federation) with an amplifier bandwidth of $35 \mathrm{~Hz}$, a time constant of $0.1 \mathrm{~s}$ and a sampling frequency of $200 \mathrm{~Hz}$. EEG absolute spectral power analysis (of at least thirty 4-s epochs) was carried out by the Fast Fourier Transform method in 8 narrow frequency sub-bands: delta (2-4 Hz), theta-1 (4-6 Hz), theta-2 (6-8 Hz), alpha-1 (8-9 Hz), alpha-2 (9-11 Hz), alpha-3 (11-13 Hz), beta-1 (13$20 \mathrm{~Hz}$ ) and beta-2 (20-30 Hz). using the "BrainSys" software ("Neurometrics", Russian Federation) [28].

\section{Statistics}

Comparison of the clinical and EEG spectral power parameters between BPD and NPD groups, as well as comparison of EEG spectral power parameters of BPD and NPD groups with the norm (HC group) was carried out using the nonparametric Mann-Whitney Utest for independent samples (with the Benjamini-Yekutieli correction for multiple comparison) using the "STATISTICA for Windows, v.10" software package. Statistical significance level was chosen not less than $\mathrm{p} \leq 0.05$ for all results presented.

\section{Results and Discussion}

\section{Clinical and psychological scores}

Studied samples of BPD and NPD patients did not differ statistically significant in values of HDRS-21 total sum, sum of points of depression cluster of HDRS-21 scale, sum of points of anxiety cluster of HDRS-21 scale, sum of points of sleep disorders cluster of HDRS-21 scale, and sum of points of ideational disorders cluster of HDRS-21 scale. As well, there were no statistically significant intergroup differences in values of HAM-A total sum. These results are presented in table 1.

\begin{tabular}{|c|c|c|c|}
\hline Clinical scores & $\begin{array}{c}\text { BPD group } \\
\mathbf{n = 2 8} \\
\text { Mean } \mathbf{2} \text { S.D. }\end{array}$ & $\begin{array}{c}\text { NPD group } \\
\mathbf{n = 2 4} \\
\text { Mean } \pm \text { S.D. }\end{array}$ & $\begin{array}{c}\text { Significance } \\
\text { of intergroup } \\
\text { differences }\end{array}$ \\
\hline $\begin{array}{c}\text { HDRS-21 scale } \\
\text { total sum }\end{array}$ & $19.7 \pm 5.1$ & $18.1 \pm 5.3$ & $\begin{array}{c}\mathrm{P}=0.30 \\
\mathrm{n} / \mathrm{s}\end{array}$ \\
\hline $\begin{array}{c}\text { HDRS-21 scale } \\
\text { depression } \\
\text { cluster }\end{array}$ & $7.0 \pm 2.2$ & $6.9 \pm 2,0$ & $\mathrm{P}=0.83$ \\
$\mathrm{n} / \mathrm{s}$ \\
$\begin{array}{c}\text { HDRS-21 scale } \\
\text { anxiety cluster }\end{array}$ & $3.4 \pm 1.8$ & $3.5 \pm 2.0$ & $\mathrm{P}=0.92$ \\
\hline $\begin{array}{c}\text { HDRS-21 scale } \\
\text { sleep disorders } \\
\text { cluster }\end{array}$ & $2.5 \pm 1.2$ & $2.2 \pm 1.5$ & $\mathrm{P}=0.89$ \\
\hline $\begin{array}{c}\text { HDRS-21 scale } \\
\text { ideational disor- } \\
\text { ders cluster }\end{array}$ & $6.0 \pm 2.0$ & $5.4 \pm 2.3$ & $\mathrm{n} / \mathrm{s}$ \\
\hline $\begin{array}{c}\text { HAM-A scale } \\
\text { total sum }\end{array}$ & $20.9 \pm 5.3$ & $18.7 \pm 7.1$ & $\mathrm{P}=0.67$ \\
$\mathrm{n} / \mathrm{s}$
\end{tabular}

Table 1: Quantitative clinical scores of BPD and NPD groups of patients.

Notes: HDRS-21 scale depression cluster - the sum of points 1, 2, 3,7 and 8 of HDRS-21 scale; HDRS-21 scale anxiety cluster - the sum of points 9, 10 and 11 of HDRS-21 scale; HDRS-21 scale sleep disorders cluster - the sum of points 4, 5 and 6 of HDRS-21 scale; HDRS-21 scale ideational disorders cluster - the sum of points 15 , 17, 19, 20 and 21 of HDRS-21 scale;

$\mathrm{n} / \mathrm{s}$ - non-significant $(\mathrm{P}>0.05)$.

\section{Intergroup EEG differences}

Topographic maps of EEG spectral power in 8 narrow frequency sub-bands, averaged over groups of patients with BPD and NPD, 
as well as of HC group, are presented at figure 1. Both groups of patients (BPD and NPD) were characterized by an organized type of EEG with predominance of the mid-frequency alpha-2 (9-11 Hz) component of the EEG alpha rhythm, which in visual EEG analysis is considered as a variant of norm.

\section{1}

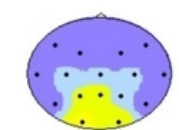
8-9 Hz

2

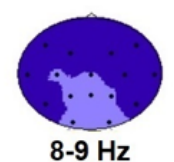

3

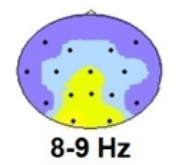

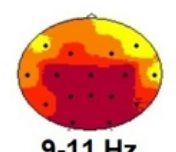
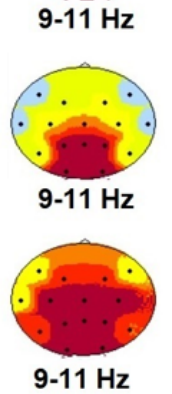

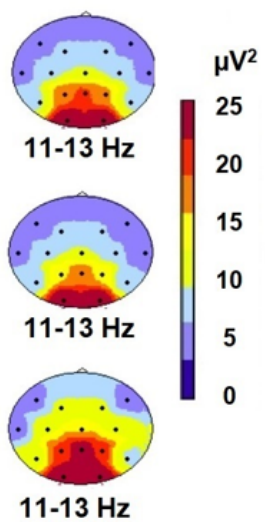

Figure 1: EEG spectral power topographic maps in 3 narrow alpha frequency sub-bands averaged by groups of patients with BPD (1), and NPD (2), and of HC (3) groups.

Notes: 1 - maps for BPD group; 2 - maps for NPD group; 3 - maps for $\mathrm{HC}$ group; EEG frequency sub-band (in $\mathrm{Hz}$ ) indicated under each map; scale to the right indicates EEG spectral power values in $\mu \mathrm{V}^{2}$.

EEG patterns in BPD and HC groups were quite similar, and the values of spectral power in all studied frequency sub-bands in all EEG leads did not differ significantly between BPD and HC groups.

In NPD group the values of spectral power of mid-frequency alpha-2 $(9-11 \mathrm{~Hz})$ and low-frequency alpha-1 $(8-9 \mathrm{~Hz})$ components of the EEG alpha rhythm were obviously lower than in BPD and HC groups. Some of these differences reached the level of statistical significance. Thus, the values of alpha-2 $(9-11 \mathrm{~Hz})$ spectral power in NPD group were significantly lower than in HC group in both occipital $(01,02)$, in both parietal (P3, P4), in both central (C3, C4), and in the left posterior temporal (T5) EEG leads (Table 2).

As well, in NPD group, compared with BPD group, spectral power values of alpha-1 $(8-9 \mathrm{~Hz})$ were significantly lower in both occipital $(01,02)$, both parietal (P3, P4), and in the right posterior temporal (T6) EEG leads. The values of spectral power of alpha-2

\begin{tabular}{|c|c|c|c|}
\hline $\begin{array}{c}\text { EEG spectral power } \\
\text { parameters } \\
\text { (EEG sub-band \& } \\
\text { EEG lead) }\end{array}$ & $\begin{array}{c}\text { NPD group } \\
\mathbf{n = 2 4} \\
\text { Mean } \pm \\
\text { S.D. } \\
\boldsymbol{\mu V}^{2}\end{array}$ & $\begin{array}{c}\text { HC group } \\
\mathbf{n = 2 4} \\
\text { Mean } \pm \text { S.D. } \\
\boldsymbol{\mu} \mathbf{V}^{2}\end{array}$ & $\begin{array}{c}\text { Significance } \\
\text { of inter- } \\
\text { group } \\
\text { differences }\end{array}$ \\
\hline Alpha-2 in 01 & $43.9 \pm 29.9$ & $\begin{array}{c}112.1 \pm \\
90.0\end{array}$ & $\mathrm{P}=0.014$ \\
\hline Alpha-2 in 02 & $51.3 \pm 39.2$ & $\begin{array}{c}126.6 \pm \\
83.9\end{array}$ & $\mathrm{P}=0.006$ \\
\hline Alpha-2 in P3 & $23.1 \pm 14.0$ & $56.0 \pm 41.3$ & $\mathrm{P}=0.007$ \\
\hline Alpha-2 in P4 & $22.8 \pm 13.4$ & $52.8 \pm 42.7$ & $\mathrm{P}=0.010$ \\
\hline Alpha-2 in C3 & $15.7 \pm 8.3$ & $28.4 \pm 12.9$ & $\mathrm{P}=0.003$ \\
\hline Alpha-2 in C4 & $14.7 \pm 8.6$ & $26.9 \pm 11.6$ & $\mathrm{P}=0.002$ \\
\hline Alpha-2 in T5 & $12.8 \pm 8.4$ & $27.8 \pm 18.7$ & $\mathrm{P}=0.014$ \\
\hline
\end{tabular}

Table 2: Differences of EEG parameters between NPD patients and $\mathrm{HC}$ group.

Notes: alpha-2 sub-band is 9-11 Hz; 01 and 02 - left and right occipital, P3 and P4 - left and right parietal, C3 and C4 - left and right central, T5 - left posterior temporal EEG leads.

(9-11 Hz) were significantly lower in both occipital $(01,02)$, in both parietal (P3, P4), in both central (C3, C4), in both temporal $(\mathrm{T} 3, \mathrm{~T} 4)$, and in the right posterior temporal (T6) EEG leads. Additionally, in NPD group the value of delta $(2-4 \mathrm{~Hz})$ spectral power in the left anterior temporal/inferior frontal EEG lead (F7) was significantly higher than in BPD group (Table 3).

Besides, in NPD group there was interhemispheric asymmetry in this brain region - the value of delta $(2-4 \mathrm{~Hz})$ spectral power in F7 was significantly higher than in F8 $\left(4.3 \mu \mathrm{V}^{2}\right.$ vs. $3.2 \mu \mathrm{V}^{2}$, respectively; $\mathrm{P}=0.019)$. The values of the interhemispheric asymmetry of the delta $(2-4 \mathrm{~Hz})$ spectral power in other EEG leads, as well as the interhemispheric asymmetry of the spectral power of the remaining EEG frequency sub-bands (theta-1, theta-2, alpha-1, alpha -2 , alpha-3, beta- 1 and beta-2) in all EEG leads in both groups of patients did not reach the level of statistical significance $(P>0.05)$.

The values of the interhemispheric asymmetry of the delta (2-4 $\mathrm{Hz}$ ) spectral power in other EEG leads, as well as the interhemispheric asymmetry of the spectral power of the remaining EEG frequency sub-bands (theta-1, theta-2, alpha-1, alpha -2 , alpha- 3 , beta- 1 and beta-2) in all EEG leads in both groups of patients did not reach the level of statistical significance $(P>0.05)$. 


\begin{tabular}{|c|c|c|c|}
\hline $\begin{array}{c}\text { EEG spectral } \\
\text { power } \\
\text { parameters } \\
\text { (EEG sub-band \& } \\
\text { EEG lead) }\end{array}$ & $\begin{array}{c}\text { BPD group } \\
\mathbf{n}=\mathbf{2 8} \\
\text { Mean } \pm \text { S.D. } \\
\mu \mathrm{V}^{2}\end{array}$ & $\begin{array}{c}\text { NPD group } \\
\mathrm{n}=24 \\
\text { Mean } \pm \text { S.D. } \\
\mu \mathrm{V}^{2}\end{array}$ & $\begin{array}{c}\text { Significance } \\
\text { of inter- } \\
\text { group } \\
\text { differences }\end{array}$ \\
\hline Alpha-1 in 01 & $13.5 \pm 9.7$ & $5.0 \pm 2.6$ & $P=0.005$ \\
\hline Alpha-1 in 02 & $12.3 \pm 9.8$ & $5.4 \pm 3.2$ & $P=0.010$ \\
\hline Alpha-1 in P3 & $10.2 \pm 7.8$ & $4.2 \pm 1.8$ & $P=0.019$ \\
\hline Alpha-1 in P4 & $9.4 \pm 7.1$ & $4.1 \pm 2.3$ & $P=0.020$ \\
\hline Alpha-1 in T6 & $5.3 \pm 4.0$ & $2.4 \pm 1.2$ & $\mathrm{P}=0.020$ \\
\hline Alpha-2 in 01 & $107.8 \pm 84.1$ & $43.9 \pm 29.9$ & $P=0.038$ \\
\hline Alpha-2 in 02 & $117.1 \pm 94.6$ & $51.3 \pm 39.2$ & $P=0.043$ \\
\hline Alpha-2 in P3 & $44.5 \pm 26.8$ & $23.1 \pm 14.0$ & $P=0.012$ \\
\hline Alpha-2 in P4 & $42.8 \pm 28.1$ & $22.8 \pm 13.4$ & $P=0.017$ \\
\hline Alpha-2 in C3 & $27.2 \pm 15.8$ & $15.7 \pm 8.3$ & $P=0.012$ \\
\hline Alpha-2 in C4 & $24.9 \pm 14.6$ & $14.7 \pm 8.6$ & $P=0.020$ \\
\hline Alpha-2 in T3 & $17.0 \pm 11.1$ & $9.6 \pm 5.7$ & $P=0.023$ \\
\hline Alpha-2 in T4 & $15.4 \pm 9.3$ & $9.0 \pm 4.7$ & $\mathrm{P}=0.027$ \\
\hline Alpha-2 in T6 & $21.3 \pm 15.1$ & $13.0 \pm 7.1$ & $P=0.050$ \\
\hline Delta in F7 & $4.0 \pm 1.5$ & $4.3 \pm 1.4$ & $P=0.049$ \\
\hline
\end{tabular}

Table 3: Differences of EEG parameters between BPD and NPD groups of patients.

Notes: alpha-1 sub-band is 8-9 Hz, alpha-2 sub-band is 9-11 Hz, delta sub-band is 2-4 Hz; 01 and 02 - left and right occipital, P3 and P4 - left and right parietal, C3 and C4 - left and right central, T3 and T4 - left and right temporal, T6 - right posterior temporal, F7 - left anterior temporal/inferior frontal EEG leads.

\section{Correlations between clinical and EEG parameters}

BPD and NPD groups of patients also differed in the structure of correlations between clinical scores and EEG spectral parameters (Table 4).

In BPD group, significant positive correlations were revealed only between the values of the sum of ideational disorders cluster (the sum of points 15, 17, 19, 20 and 21 of HDRS-21 scale) and the values of EEG alpha-2 (9-11 Hz) spectral power in frontal, central and temporal regions (in F3, F4, F8, C3, C4, T3 and T4 EEG leads).

In NPD group, significant negative correlations were revealed only between the values of the total sum of HAM-A scale and the values of EEG alpha-2 (9-11 Hz) spectral power in frontal, central and temporal regions: in F7, F3, F4, F8, C3, C4, T3 and T4 EEG leads.
The results obtained indicate a high informativeness of the quantitative EEG in relation to the identification of subtle differences in the brain functional state between the groups of patients with BPD and NPD, despite the fact that both these groups belong to the same demonstrative cluster $\mathrm{B}$ of personality disorders, and did not differ in clinical scores. As well, EEG differences were found between NPD group and norm (HC group).

The absence of statistically significant differences between the EEG spectral parameters of BPD and HC groups coincides with some literature data [16].

Significantly lower values of EEG alpha-2 (9-11 Hz) spectral power in NPD group compared to norm (HC group), and of both alpha-1 (8-9 Hz) and alpha-2 (9-11 Hz) spectral power compared to BPD group, indicate that brain cortex in NPD patients is more activated than in BPD and HC groups. A possible cause of this inhibitory deficit in NPD group may be a decreased functional state of the anterior cortical regions (especially of the left hemisphere). It is reflected in higher values of EEG delta spectral power in the projection of the left dorsolateral prefrontal cortex (in F7 EEG lead) in NPD group compared to BPD group. This brain area is a powerful source of descending inhibitory influences on other cortical areas and subcortical structures [29]. The EEG data obtained in this study are consistent with MRI data on the gray matter abnormalities of the prefrontal cortex in NPD patients [15]. Decreased functional state of the anterior cortical regions causes a deficit in descending inhibition underlying dysregulation of emotions and increased impulsivity in NPD patients, that may entail disturbances in the control of their behavior and provokes auto-aggressive actions up to suicidal attempts $[29,30]$.

In BPD group, significant positive correlations were revealed only between the values of the sum of ideational disorders cluster and the values of EEG alpha-2 $(9-11 \mathrm{~Hz})$ spectral power in frontal, central and temporal regions. Thus, more pronounced ideational disorders are associated with relatively less activation of these brain areas. In NPD group, significant negative correlations were revealed only between the values of the total sum of HAM-A scale and the values of EEG alpha-2 $(9-11 \mathrm{~Hz})$ spectral power in frontal, central and temporal regions. So, less activation (more alpha) of these regions is associated with lower anxiety. Such structure of correlations between clinical and EEG parameters in BPD and NPD 


\begin{tabular}{|c|c|c|c|}
\hline & BPD group $(n=28)$ & & \\
\hline Clinical scores & $\begin{array}{l}\text { EEG spectral power parameters } \\
\text { (EEG sub-band \& EEG lead) }\end{array}$ & $\begin{array}{c}\text { Spearman's } \\
\mathbf{r}\end{array}$ & Significance \\
\hline \multirow{8}{*}{$\begin{array}{l}\text { Sum of ideational disorders } \\
\text { cluster of HDRS-21 scale }\end{array}$} & Alpha-2 in F3 & 0.54 & $P=0.005$ \\
\hline & Alpha-2 in F4 & 0.50 & $P=0.011$ \\
\hline & Alpha-2 in F8 & 0.45 & $P=0.025$ \\
\hline & Alpha-2 in C3 & 0.41 & $P=0.041$ \\
\hline & Alpha-2 in C4 & 0.49 & $P=0.014$ \\
\hline & Alpha-2 in T3 & 0.45 & $P=0.023$ \\
\hline & Alpha-2 in T4 & 0.44 & $P=0.028$ \\
\hline & NPD group $(n=24)$ & & \\
\hline Clinical scores & $\begin{array}{l}\text { EEG spectral power parameters } \\
\text { (EEG sub-band \& EEG lead) }\end{array}$ & $\begin{array}{c}\text { Spearman's } \\
\text { r }\end{array}$ & Significance \\
\hline \multirow[t]{8}{*}{ HAM-A scale total sum } & -Alpha-2 in F7 & -0.42 & $P=0.044$ \\
\hline & -Alpha-2 in F3 & -0.44 & $P=0.035$ \\
\hline & -Alpha-2 in F4 & -0.52 & $P=0.011$ \\
\hline & -Alpha-2 in F8 & -0.51 & $P=0.013$ \\
\hline & -Alpha-2 in C3 & -0.47 & $P=0.023$ \\
\hline & -Alpha-2 in C4 & -0.54 & $P=0.007$ \\
\hline & -Alpha-2 in T3 & -0.44 & $P=0.034$ \\
\hline & -Alpha-2 in T4 & -0.44 & $P=0.037$ \\
\hline
\end{tabular}

Table 4: Correlations between clinical and EEG parameters in BPD and NPD groups of patients.

Notes: sum of ideational disorders cluster - the sum of points 15, 17, 19, 20 and 21 of HDRS-21 scale; alpha-2 sub-band is 9-11 Hz; F7 and F8 - left and right anterior temporal/inferior frontal, F3 and F4 - left and right frontal, C3 and C4 - left and right central, T3 and T4 - left and right temporal EEG leads; the sign - (minus) indicates negative correlations.

groups of patients suggests that EEG pattern in BPD group reflects mainly the ideational aspects of disorder, while in NPD group it reflects mainly the emotional aspects of disorder.

Limitations of the study are determined by the relatively small number of patients of only male gender that requires, in the future, an increase in the size and balance of the gender composition of the samples.

\section{Conclusion}

The EEG data obtained confirmed some differences in the brain functional state between the groups of patients with BPD and NPD. EEG spectral parameters of BPD groups do not differ from norm. In NPD patients, brain cortex is more activated than in BPD and norm groups. A possible cause of this inhibitory deficit is a decreased functional state of the anterior cortical regions. The differences in structure of correlations between clinical and EEG parameters in BPD and NPD groups of patients suggests that EEG pattern in BPD group reflects mainly the ideational aspects of disorder, while in NPD group it reflects mainly the emotional aspects of disorder. The noted features of the functional organization of the brain activity may contribute to the disturbance of the processes of regulation of emotions and control of behavior in adolescent patients with personality disorders, more pronounced in narcissistic personality disorder.

\section{Acknowledgements}

No.

\section{Conflict of Interest}

The authors have no potential conflicts of interest to disclose. 


\section{Author Contributions}

- $\quad$ Conceptualization: Ekaterina V. Iznak, Elena S. Krylova, Vasiliy G. Kaleda, Andrey F. Iznak.

- Data curation: Ekaterina V. Iznak, Elena V. Damyanovich, Elena S. Krylova, Alexey A. Kuleshov.

- $\quad$ Formal analysis: Ekaterina V. Iznak, Elena S. Krylova, Alexey A. Kuleshov, Andrey F. Iznak.

- Investigation: Ekaterina V. Iznak, Elena V. Damyanovich, Elena S. Krylova, Alexey A. Kuleshov, Andrey F. Iznak.

- $\quad$ Methodology: Ekaterina V. Iznak, Elena S. Krylova, Vasiliy G. Kaleda, Andrey F. Iznak.

- Supervision: Vasiliy G. Kaleda, Andrey F. Iznak.

- Validation: Ekaterina V. Iznak, Elena S. Krylova, Vasiliy G. Kaleda, Andrey F. Iznak.

- Visualization: Ekaterina V. Iznak, Andrey F. Iznak.

- Writing—original draft: Ekaterina V. Iznak, Elena S. Krylova, Alexey A. Kuleshov, Vasiliy G. Kaleda, Andrey F. Iznak.

- Writing-review and editing: all authors.

\section{Bibliography}

1. Sharp C and Fonagy P. "Practitioner Review: Borderline personality disorder in adolescence - Recent conceptualization, intervention, and implications for clinical practice". Journal of Child Psychology and Psychiatry and Allied Disciplines 56.12 (2015): 1266-1288.

2. Ha C., et al. "Psychiatric comorbidity in hospitalized adolescents with borderline personality disorder". The Journal of Clinical Psychiatry 75.5 (2014): e457-464.

3. Kaess M., et al. "Borderline personality disorder in adolescence". Pediatrics 134.4 (2014): 782-793.

4. Cain NM and Boussi A. "Narcissistic Personality Disorder". In: Zeigler-Hill V., and Shackelford TK, eds. "Encyclopedia of Personality and Individual Differences". Springer International Publishing. Pages (2020): 3088-3096.

5. Soloff PH and Chiappetta L. "Subtyping borderline personality disorder by suicidal behavior". Journal of Personality Disorders ;26.3 (2012): 468-480.
6. Coleman D., et al. "Narcissistic personality disorder and suicidal behavior in mood disorders". Journal of Psychiatric Research 85.1 (2017): 24-28.

7. Krylova ES., et al. "Non-suicidal self-injury and its relation to suicidal behavior in youth patients with personality disorders". Suicidology 1 (2019): 48-57.

8. Krylova ES., et al. "Auto-aggressive behavior in patients with narcissistic and borderline personality disorder in youth". Psikhicheskoe zdorovie [Mental Health];12 (2020): 37-47.

9. Brown RC and Plener PL. "Non-suicidal self-injury in adolescence". Current Psychiatry Reports 19.3 (2017): 1-8.

10. Colle L., et al. "Self-Harming and sense of agency in patients with borderline personality disorder". Frontiers in Psychiatry 11.1 (2020): 1-11.

11. Dawood S., et al. "Pathological narcissism and nonsuicidal selfinjury". Journal of Personality Disorders 32.1 (2018): 87-108.

12. Links PS and Eynan R. "The relationship between personality disorders and axis I psychopathology: Deconstructing comorbidity". Annual Review of Clinical Psychology 9 (2013): 529554.

13. Brunner R., et al. "Neurobiological findings in youth with borderline personality disorder". Scandinavian Journal of Child and Adolescent Psychiatry and Psychology 3.1 (2015): 22-30.

14. Brunner R., et al. "Reduced prefrontal and orbitofrontal gray matter in female adolescents with borderline personality disorder: is it disorder specific?" Neuroimage 49.1 (2010): 114120.

15. Schulze L., et al. "Gray matter abnormalities in patients with narcissistic personality disorder". Journal of Psychiatric Research 47.10 (2013): 1363-1369.

16. Pop-Jordanova N., et al. "Analysis of EEG Characteristics and Coherence in Patients Diagnosed as Borderline Personality". Pril (Makedon Akad Nauk Umet Odd Med Nauki) 40.3 (2019): 57-68.

17. Russ MJ., et al. "EEG theta activity and pain insensitivity in self-injurious borderline patients". Psychiatry Research 89.3 (1999): 201-214. 
18. Lee SM., et al. "Electroencephalographic Correlates of Suicidal Ideation in the Theta Band". Clinical EEG and Neuroscience 48.5 (2017): 316-321.

19. Knyazev GG. "EEG correlates of personality types”. Netherlands Journal of Psychology. 62.1 (2006): 78-87.

20. Smith EE., et al. "Assessing and conceptualizing frontal EEG asymmetry: An updated primer on recording, processing, analyzing, and interpreting frontal alpha asymmetry". International Journal of Psychophysiology 111 (2017): 98-114.

21. Beeney JE., et al. "EEG asymmetry in borderline personality disorder and depression following rejection". Personality Disorders 5.2 (2014): 178-185.

22. Moshirian Farahi SM., et al. "Neuroticism and Frontal EEG Asymmetry Correlated with Dynamic Facial Emotional Processing in Adolescents". Frontiers in Psychology 10 (2019): 175.

23. Iznak EV., et al. "EEG Asymmetries in Depressive Female Adolescents with Different Kinds of Auto-Aggressive Behavior". Psychiatry (Moscow) 18.3 (2020): 14-21.

24. Iznak AF., et al. "Differences of EEG Frequency and Spatial Parameters in Depressive Female Adolescents with Suicidal Attempts and Non-suicidal Self-injuries". Clinical EEG and Neuroscience 52.6 (2021): 406-413.

25. First MB., et al. "Structured clinical interview for DSM-5 personality disorders (SCID-5-PD)". Washington: American Psychiatric Association Publishing (2016).

26. Hamilton M. "A rating scale for depression". Journal of Neurology. Neurosurgery and Psychiatry 23 (1960): 56-62.

27. Hamilton M. "The assessment of anxiety states by rating". British Journal of Medical Psychology 32 (1959): 50-55.

28. Mitrofanov AA. "Computer system for analysis and topographic mapping of the brain electrical activity with a neurometric EEG data bank (description and application)". Moscow (2005): 63.

29. Forbes CE., et al. "The role of executive function and the dorsolateral prefrontal cortex in the expression of neuroticism and conscientiousness". Social Neuroscience 9 (2014): 139-151.
30. Lang S., et al. "Cortical inhibition in alexithymic patients with borderline personality disorder". Biological Psychology 88.2 (2011): 227-232.

\section{Assets from publication with us}

- Prompt Acknowledgement after receiving the article

- Thorough Double blinded peer review

- Rapid Publication

- Issue of Publication Certificate

- High visibility of your Published work

Website: www.actascientific.com/

Submit Article: www.actascientific.com/submission.php

Email us: editor@actascientific.com

Contact us: +919182824667 